\title{
ABSTRACT REVIEW COMMITTEE
}

\section{CHAIRMAN}

\section{Dr. Mona AlDabbagh}

Consultant, Pediatric Infectious Diseases and Transplant Infectious Diseases

Department of Pediatrics

King Abdulaziz Medical City, Ministry of National Guard Health Affairs-WR

Head, Research Training and Development- King Abdullah International Medical Research Center-WR

\section{MEMBERS}

Dr. Jamilah AIRahimi

Consultant, Cardiologist

Deputy Chairman, King Faisal Cardiac Center, King Faisal Cardiac Center King

Abdulaziz Medical City, Ministry of National Guard Health Affairs-WR Director, Post

Graduate Medical Education

King Saud bin Abdulaziz University for Health Sciences

\section{Dr. Amr AlBanna}

Consultant Pulmonologist

Deputy Chairman, Quality and Patient Safety, Department of Medicine King Abdulaziz Medical City, Ministry of National Guard Health Affairs-WR Head of

Research Office- King Abdullah International Medical Research Center-WR

\section{Dr. Areej AlOwfi}

Consultant, Family Medicine

Department of Family Medicine

King Abdulaziz Medical City, Ministry of National Guard Health

Affairs-WR Associate Dean, University Pre-Professional Program,

Jeddah - Female Branch

King Saud bin Abdulaziz University for Health Sciences

\section{Dr. Majed AlThaqafy}

Consultant Public Health \& Epidemiology

Department of Infection Prevention \& Control

King Abdulaziz Medical City, Ministry of National Guard Health Affairs-WR
Dr. Taghrid AlDosari

Oral Biologist

Community and Preventive Medicine Department

King Abdulaziz Medical City, Ministry of National Guard Health Affairs-WR

Dr. Sherine Ismail

Nephrology / Transplant Clinical Pharmacist

Department of Pharmaceutical Care Services

King Abdulaziz Medical City, Ministry of National Guard Health Affairs-WR

Dr. Mohammed Azfar Qureshi

Nephrology Staff Physician Department of Medicine

King Abdulaziz Medical City, Ministry of National Guard Health Affairs-WR

Mr. Mohammad AlZahrani

Research Coordinator, Bioethics Department

King Abdullah International Medical Research Center-WR

Ms. Duaa Olwi

Pre-Scholar Epidemiologist

King Abdullah International Medical Research Center-WR

Ms. Ebtisam Jan

Education Coordinator, Research Training and Development

King Abdullah International Medical Research Center-WR 